\title{
VARIÉTÉS KÄHLERIENNES ET PREMIÈRE CLASSE DE CHERN
}

\author{
ANDRE LICHNEROWICZ
}

\section{Introduction}

Dans deux intéressants articles [3], [4], S. Kobayashi a étudié les variétés kähleriennes compactes $W_{n}$ admettant une première classe de Chern définie positive ou définie négative. Le but du présent article est d'obtenir certaines propriétés des tenseurs holomorphes de $W_{n}$ (contravariants ou covariants) sous des hypothèses plus faibles concernant $c_{1}\left(W_{n}\right)$ (caractère non positif ou non négatif). On en déduit en particulier des propriétés du plus grand groupe connexe de transformations holomorphes de $W_{n}$ (voir [9]).

Les principaux résultats sont énoncés dans les théorèmes 1 et 2 ( $\S 11$ et 12) et constituent, en un certain sens, une extension de résultats de Nakano, Kobayashi et Kodaira.

Les lemmes 1, 2 et 3 donnent les principaux instruments permettant d'établir ces théorèmes qui font intervenir aussi la proposition $2 \mathrm{du} \S 10$. Celle-ci fournit une condition nécessaire et suffisante commode, en termes d'opérateurs introduits par Kodaira, pour qu'un tenseur antisymétrique (contravariant) soit holomorphe. Certains des résultats ont été énoncés dans [10].

Dans un but de simplicité, on a regroupé dans les $\S 1$ à 3 certaines des notations et des formules utilisées.

\section{Variété complexes}

a) $W_{n}$ étant une variété différentiable connexe, paracompacte, de dimension réelle $2 n$, supposons qu'elle admette une structure analytique complexe. Un système de coordonnées locales complexes est défini dans un domaine $U$ de $W_{n}$ par:

$$
\phi_{U}: z \in U \rightarrow\left\{z^{\alpha}\right\} \in C^{n} \quad(\alpha=1, \cdots, n) .
$$

Nous posons $z^{\alpha}=\bar{z}^{\alpha}(\bar{\alpha}=n+1, \cdots, 2 n)$ et désignons par $\left\{z^{k}\right\}(k=1, \cdots, 2 n)$ l'ensemble des $2 n$ nombres complexes $\left\{z^{\alpha}, z^{\alpha}\right\}$. Dans l'intersection $U \cap V$ des domaines de deux systèmes de coordonnées complexes, les coordonnées complexes $\left\{z^{\alpha}\right\}$ de $z$ considéré comme appartenant à $U$, sont des fonctions 
holomorphes, à jacobien $\mathscr{J}_{V}^{U}$ non nul, des coordonnées complexes $\left\{z^{\lambda^{\prime}}\right\}$ du même point $z$ considéré comme appartenant à $V$. La structure complexe munit $W_{n}$ d'une orientation naturelle.

On sait que la structure complexe de $W_{n}$ détermine en chaque point $z$ de la variété une structure complexe de l'espace vectoriel tangent $T_{z}$ qui peut être définie soit par un sous-espace complexe convenable $S_{z}^{c}$, de dimension complexe $n$, du complexifié $T_{z}^{c}$ de $T_{z}\left(S_{z}^{c} \cap \bar{S}_{z}^{c}=0\right)$, soit par l'opérateur $J_{z}$, de carré-Id, défini de la maniè re suivante: si $v$ de composantes $v^{\alpha}, v^{\alpha}$ appartient à $T_{z}^{c}$, on a :

$$
\left(J_{z} v\right)^{\alpha}=i v^{\alpha}, \quad\left(J_{z} v\right)^{\alpha}=-i v^{\alpha} .
$$

Le champ $J$ des opérateurs $J_{z}$ définit la structure "presque complexe" de $W_{n}$ déterminée par sa strutcture complexe; $S_{z}^{c}$ et $\bar{S}_{z}^{c}$ sont les deux sous espaces propres de $J_{z}$ et

$$
T_{z}^{c}=S_{z}^{c} \oplus \bar{S}_{z}^{c}
$$

b) L'existence de cette décomposition-ou si l'on préfère celle d'indices de deux espèces-conduit à la notion de type pour un tenseur et pour un opérateur.

Nous appelons p-forme une forme différentielle extérieure à valeurs complexes de degré $p$. Par abréviation, nous appelons $p$-tenseur un tenseur contravariant antisymétrique complexe d'ordre $p$.

Soit $d$ l'opérateur de différentiation extérieure sur les formes. Nous posons ici $d=d^{\prime}+d^{\prime \prime}$, où $d^{\prime}$ est de type $(1,0)$ et $d^{\prime \prime}$ de type $(0,1)$. De $d^{2}=0$, on déduit par considération des types:

$$
d^{\prime 2}=0, \quad d^{\prime \prime 2}=0, \quad d^{\prime} d^{\prime \prime}+d^{\prime \prime} d^{\prime}=0 .
$$

Une $p$-forme holomorphe est une $p$-forme $\beta$ de type $(p, 0)$ telle que $d^{\prime \prime} \beta$ $=0$. Il est équivalent de dire que c'est une forme de type $(p, 0)$ qui, pour tout voisinage $U$ de coordonnées locales complexes, a pour coefficients des fonctions holomorphes dans $U$ des $z^{\alpha}$.

Un p-tenseur holomorphe est un $p$-tenseur $A$ de type $(p, 0)$ qui, pour tout voisinage $U$ de coordonnées locales complexes, a pour composantes des fonctions holomorphes dans $U$ des $z^{\alpha}$.

c) Une transformation holomorphe de $W_{n}$ est une transformation de $W_{n}$ laissant invariante sa structure complexe, ou-ce qui est équivalent-laissant $J$ invariant. Une transformation infinitésimale (t.i.) définie par un champ de vecteurs $X$ est une t.i. holomorphe si:

$$
\mathscr{L}(X) J=0
$$

où $\mathscr{L}(X)$ est l'opérateur de transformation infinitésimale (ou de dérivation de Lie). En coordonnées locales complexes, (1.3) s'écrit: 


$$
\partial_{\beta} X^{\alpha}=0, \quad\left(\partial_{\alpha}=\frac{\partial}{\partial z^{\alpha}}, \quad \partial_{\beta}=\frac{\partial}{\partial z^{\beta}}\right) .
$$

$\left(1.3^{\prime}\right)$ exprime que $X^{(1,0)}$, partie de type $(1,0)$ de $X$, est un 1-tenseur ou vecteur holomorphe. Si $X$ définit une t.i. holomorphe, il en est de même pour $J X$ et l'on a:

$$
[J X, Y]=[X, J Y]=J[X, Y] .
$$

Dans la suite $W_{n}$ est suposée compacte.

Soit $G$ le plus grand groupe connexe de transformations holomorphes de $W_{n}$ compacte. Bochner et Montgomery ont établi que $G$ est un groupe de Lie complexe opérant sur $W_{n}$ de manière analytique complexe ou holomorphe. L'algèbre de Lie de $G$ peut être identifié à l'algèbre $L_{h}$ des transformations infinitésimales holomorphes. D'après (1.4), $J$ définit sur $L_{h}$ une structure d'algèbre de Lie complexe et celle-ci correspond à la structure complexe de $G$.

\section{L'ideal I de $\boldsymbol{L}_{h}$}

a) Si $h$ est une 1 -forme holomorphe de $W_{n}, X$ un élément de $L_{h}$, le scalaire $X^{\alpha} h_{\alpha}$ est holomorphe sur la variété compacte $W_{n}$. Par suite:

$$
i(X) h=X^{\alpha} h_{\alpha}=\text { const. }
$$

où $i(X)$ désigne l'opérateur de produit intérieur par $X$.

Soit $H^{(1)}$ l'espace vectoriel complexe des 1-formes holomorphes fermées. On sait que toute forme $h \in H^{(1)}$ à périodes imaginaires pures est nécessairement nulle. Si $b_{p}\left(W_{n}\right)$ désigne le $p^{e}=$ nombre de Betti de la variété, on a donc $\operatorname{dim}_{R} H^{(1)} \leqslant b_{1}\left(W_{n}\right)$. Nous désignons par $H_{0}^{(1)}$ le sous-espace de $H^{(1)}$ défini par les éléments $h$ de $H^{(1)}$ tels que $i(X) h=0$ pour tout $X \in L_{h}$.

b) Soit $I$ l'espace vectoriel complexe défini par les $X \in L_{h}$ tels que:

$$
i(X) h=0 \quad \text { pour tout } h \in H^{(1)} .
$$

Si $X, Y \in L_{h}$ considérons le crochet $Z=[X, Y]$ et le produit:

$$
i(\mathcal{Z}) h=Z^{\alpha} h_{\alpha}=X^{\beta} \partial_{\beta} Y^{\alpha} h_{\alpha}-Y^{\beta} \partial_{\beta} X^{\alpha} h_{\alpha}
$$

soit:

$$
i(Z) h=X^{\beta} \partial_{\beta}\left(Y^{\alpha} h_{\alpha}\right)-Y^{\beta} \partial_{\beta}\left(X^{\alpha} h_{\alpha}\right)-X^{\alpha} Y^{\beta}\left(\partial_{\alpha} h_{\beta}-\partial_{\beta} h_{\alpha}\right)=0 .
$$

Ainsi si $L_{h}^{\prime}=\left[L_{h}, L_{h}\right]$ est l'idéal dérivé de $L_{h}$, on a $L_{h}^{\prime} \subset I$ et $I$ est un idéal de $L_{h}$ tel que $L_{h} / I$ soit abélien [9].

La forme bilinéaire $i(X) h$ (où $X \in L_{h}, h \in H^{(1)}$ ) met en dualité les espaces vectoriels $L_{h} / I$ et $H^{(1)} / H_{0}^{(1)}$. Par suite: 


$$
\operatorname{dim}_{C} L_{h}-\operatorname{dim}_{C} I=\operatorname{dim}_{C} H^{(1)}-\operatorname{dim}_{C} H_{0}^{(1)} .
$$

\section{Variétés Kähleriennes}

a) Dans toute la suite, nous supposons que la variété compacte $W_{n}$ considérée admet une métrique kählérienne qui s'écrit localement

$$
d s^{2}=2 g_{\alpha \beta} d z^{\alpha} d z^{\bar{\beta}}
$$

la 2-forme (réelle) fondamentale associée étant:

$$
F=i g_{\alpha \beta} d z^{\alpha} \wedge d z^{\beta}
$$

Pour que la métrique hermitienne (3.1) soit kählérienne, il faut et il suffit que la 2-forme $F$ soit fermée. Elle est alors à dérivée covariante nulle dans la connexion riemannienne définie par la métrique [8]. En coordonnées locales complexes, les seuls coefficients non nécessairement nuls de cette connexion sont ceux de types purs:

$$
\Gamma_{\beta \tau}^{\alpha}, \quad \Gamma_{\bar{\beta} \bar{\tau}}^{\alpha}=\bar{\Gamma}_{\beta_{\tau}}^{\alpha} .
$$

Soit $A$ un $p$-tenseur de type $(p, 0)$. A un tel $p$-tenseur correspond de manière naturelle, par dualité définie par la métrique et conjugaison, une $p$-forme notée $\alpha(A)$ de type $(p, 0)$ avec:

$$
{\overline{(\alpha(A))_{p_{1} \cdots p_{p}}}}=g_{p_{1} \sigma_{1}} \cdots g_{p_{p} \sigma_{p}} A^{\sigma_{1} \cdots \sigma_{p}} \text {. }
$$

Inversement toute forme de type $(p, 0)$ est l'image par $\alpha$ d'un $p$-tenseur de type $(p, 0)$.

Nous notons $(\alpha, \beta)$ le produit intérieur de deux $p$-formes considéré comme une fonction du point $z \in W_{n}$, par $\langle\alpha, \beta\rangle$ le produit scalaire global

$$
\langle\alpha, \beta\rangle=\int_{W_{h}}(\alpha, \beta) \eta,
$$

où $\eta$ est la forme élément de volume définie par la métrique et l'orientation de $W_{n}$.

b) Soit $\delta$ l'opérateur de codifférentiation sur les formes qui s'exprime immédiatement, au signe près, par la dérivation covariante contractée. On pose $\delta=\delta^{\prime}+\delta^{\prime \prime}$, où $\delta^{\prime}$ est de type $(-1,0)$, et $\delta^{\prime \prime}$ de type $(0,-1)$. De $\delta^{2}=0$, on déduit encore:

$$
\delta^{\prime 2}=0, \quad \delta^{\prime 2}=0, \quad \delta^{\prime} \delta^{\prime \prime}+\delta^{\prime \prime} \delta^{\prime}=0 .
$$

On sait que $\delta$ est transposé de $d$ par le produit scalaire introduit. De même $\delta^{\prime}$ est transposé de $d^{\prime}$ et $\delta^{\prime \prime}$ de $d^{\prime \prime}$, ce qui se traduit par les relations: 


$$
\left\langle d^{\prime} \alpha, \beta\right\rangle=\left\langle\alpha, \delta^{\prime} \beta\right\rangle, \quad\left\langle d^{\prime \prime} \alpha, \beta\right\rangle=\left\langle\alpha, \delta^{\prime \prime} \beta\right\rangle .
$$

Le laplacien $\Delta=d \delta+\delta d$ de G. de Rham sur les formes admet aussi, sur une variété kählerienne les expressions:

$$
\Delta=2\left(d^{\prime} \delta^{\prime}+\delta^{\prime} d^{\prime}\right)=2\left(d^{\prime \prime} \delta^{\prime \prime}+\delta^{\prime \prime} d^{\prime \prime}\right) .
$$

Soit enfin $M$ l'opérateur linéaire sur les formes défini sur les formes de type $(p, q)$ par la relation:

$$
M \alpha_{p, q}=(p-q) i \alpha_{p, q} .
$$

On établit [8] que cet opérateur satisfait aux relations de commutation:

$$
\delta M-M \delta=d \Lambda-\Lambda d, \quad d M-M d=-[\delta L-L \delta]=-i\left(d^{\prime}-d^{\prime \prime}\right),
$$

où $L$ (resp. $\Lambda$ ) sont les opérateurs de produit extérieur (resp. intérieur) pour la forme $F$. Il en résulte aisément que $\Delta$ commute avec les opérateurs $L$ et $A$.

c) Soit $H^{(p)}$ l'espace complexe des p-formes holomorphes de $W_{n}$; nous désignons par $b_{p, 0}\left(W_{n}\right)$ sa dimension complexe.

Si $\beta \in H^{(p)}$, elle vérifie $d^{\prime \prime} \beta=0$ et aussi $\delta^{\prime \prime} \beta=0$ pour une raison de type; $\beta$ est par suite harmonique au sens de $\Delta$ d'après (3.4), donc fermée $(d \beta=0)$ et cofermée $(\delta \beta=0)$. Ainsi sur une variété kählerienne, toute $p$-forme holomorphe est fermée et même harmonique.

Il en est en particulier ainsi pour les 1 -formes holomorphes qui sont intervenues au $\S 2$. On voit de plus que $b_{1}\left(W_{n}\right)=2 b_{1,0}\left(W_{n}\right)$.

d) Soit $A$ un $p$-tenseur holomorphe sur $W_{n}$. On a:

$$
\partial_{\beta} A^{\rho_{1} \cdots \rho_{p}}=\nabla_{\beta} A^{\rho_{1} \cdots \rho_{p}}=0,
$$

où $V$ est l'opérateur de dérivation covariante dans la connexion riemanienne. Par passage à la forme $\alpha(A)$, les relations (3.6) se traduisent par:

$$
\nabla_{\beta} \alpha(A)_{\rho_{1} \ldots \rho_{p}}=0 .
$$

Par antisymétrisation de (3.7), on voit que $\alpha(A)$ est nécessairement $d^{\prime}$-fermée $\left(d^{\prime} \alpha(A)=0\right)$. On sait que $d^{\prime}$ (ou $d^{\prime \prime}$ ) définit une cohomologie localement triviale. Du théorème de décomposition de Hodge-de Rham il résulte que l'on peut décomposer $\alpha(A), d^{\prime}$-fermée, selon:

$$
\alpha(A)=d^{\prime} \beta+H \alpha(A),
$$

où $\beta$ est une (p-1)-forme et où $H \alpha(A)$ est une $p$-forme holomorphe. Le scalaire holomorphe défini par le produit intérieur $(\alpha(A), H \alpha(A))$ est une constante et nous posons: 


$$
k(A)=(\alpha(A), H \alpha(A)) .
$$

On a par orthogonalité:

$$
<H \alpha(A), H \alpha(A)>=\langle\alpha(A), H \alpha(A)\rangle=k(A) \int_{W_{n}} \eta .
$$

Ainsi pour que $k(A)=0$, il faut et il suffit que $H \alpha(A)=0$ c'est-à-dire que $\alpha(A)$ soit $d^{\prime}$-cohomologue à zéro.

Considérons en particulier le cas $p=1$, c'est-à-dire le cas des t.i. holomorphes $X$. Nous pouvons les définir à partir des 1-formes $\xi=\alpha\left(X^{(1,0)}\right)$, de type $(1,0)$, vérifiant $\nabla_{\beta} \xi_{\alpha}=0$. La décomposition (3.8) s'écrit dans ce cas

$$
\xi=d^{\prime} \rho+H \xi \text {, }
$$

où $\rho$ est un scalaire complexe. Pour que $X \in I$, il faut et il suffit que $k\left(X^{(1,0)}\right)=$ $i(X) H \xi=0$, c'est-à-dire que $\xi$ vérifie $\nabla_{\beta} \xi_{\alpha}=0$ et soit $d^{\prime}$ cohomologue à zéro [9].

\section{Caractère positif ou nul d'une classe de cohomoloqie de type (1.1)}

a) Soit $\gamma$ une 2 -forme réelle de type $(1,1)$. A cette 2 -forme qui s'écrit localement

$$
\gamma=\gamma_{\alpha \beta} d z^{\alpha} \wedge d z^{\beta}
$$

associons la forme hermitienne $c$ de composantes

$$
c_{\alpha \beta}=-i \gamma_{\alpha \beta} .
$$

En particulier à la 2-forme $F$ de $W_{n}$ se trouve ainsi associée la métrique de $W_{n}$. Nous écrirons $\gamma \geqslant 0$ (resp $\left.\leqslant 0\right)$ si la forme hermitienne $c$ associée à $\gamma$ est positive ou nulle (resp. négative ou nulle) aux différents points de $W_{n}$.

b) Supposons $\gamma$ fermée et notons $[\gamma]$ sa classe de cohomologie réelle. $D$ 'après la classique décomposition en classes primitives par rapport à la classe fondamentale $[F]$, on a:

$$
[\gamma]=a[F]+[\beta] \quad(\Lambda[\beta]=0)
$$

(où $a$ est une constante réelle) ou en termes de formes:

$$
\gamma=a F+\beta+d \mu
$$

avec $\Lambda \beta=0$ et $\mu, 1$-forme. Par produit par $\Lambda$, il vient:

$$
\Lambda \gamma=a n+\Lambda d \mu \text {. }
$$


Mais d'après (3.5)

$$
d \Lambda \mu-\Lambda d \mu=\delta M \mu-M \delta \mu
$$

où $d \Lambda \mu=M \delta \mu=0$ pour des raisons de degré. Ainsi :

$$
A_{\gamma}=a_{n}-\delta M \mu \text {. }
$$

Par intégration sur $W_{n}$, il vient :

$$
a n \int_{W_{n}} \eta=\int_{w_{n}}(\Lambda \gamma) \eta .
$$

Si $\gamma$, non identiquement nulle, est $\geqslant 0, \Lambda_{\gamma}$ est $\geqslant 0$ et non identiquement nul. On a donc $a>0$ et, d'après (4.1), la classe $[\gamma]$ de la forme envisagée est certainement $\neq 0$. Il en résulte qu'une classe de cohomologie non nulle de type $(1,1)$ ne peut contenir simultanément une forme $\geqslant 0$ et une forme $\leqslant 0$.

Nous dirons qu'une classe de type $(1,1)$ est $\geqslant 0$ (resp $\leqslant 0$ ) si elle contient une forme fermée $\geqslant 0$ (resp. $\leqslant 0$ ). Une classe à la fois $\geqslant 0$ et $\leqslant 0$ est certainement nulle.

c) Nous notons $c_{1}\left(W_{n}\right)$ la première classe de Chern c'est-à-dire celle de degré 2-de la variété $W_{n}$. Soit $R$ le tenseur de Ricci de la variété, dont nous désignons par $R_{\alpha \beta}$ les composantes en coordonnées locales complexes dans un domaine $U$. Si $g_{U}$ désigne le descriminant dans $U$ de la métrique:

$$
R_{\alpha \beta}=-\partial_{\alpha} \partial_{\beta} \log \sqrt{g_{U}} .
$$

On sait que $c_{1}\left(W_{n}\right)$ peut être définie par une 2-forme réelle fermée $\tau$, à périodes entières, qui ne diffère de la 2-forme associée à $R$ que par un facteur de normalisation. Plus précisément sur $U$ :

$$
\tau=(2 \pi)^{-1} i R_{\alpha \beta} d z^{\alpha} \wedge d z^{\beta} .
$$

Nous nous intéressons ici aux cas où $c_{1}\left(W_{n}\right) \geqslant 0$ et $c_{1}\left(W_{n}\right) \leqslant 0$, c'est-à-dire au cas où la classe de cohomologie réelle définie par $c_{1}\left(W_{n}\right)$ est positive ou nulle, ou bien négative ou nulle. Il en est ainsi pour un certain nombre de variétés algébriques intéressantes.

Les cas où $c_{1}\left(W_{n}\right)$ contient une forme partout définie positive ou définie négative ont été étudiés par de nombreux auteurs, en particulier Kodaira, Nakano, Andreotti et S. Kobayashi [4], [5]. Une fraction notable de leurs résultats se laisse généraliser de manière convenable aux hypothèses faites ici.

\section{Interprétation de $c_{1}\left(W_{n}\right)=0$}

a) Rappelons à quelle condition une 2-forme réelle fermée $\gamma$ de type $(1,1)$ 
est homologue à 0 . Il existe alors une 1 -forme réelle $\lambda$ telle que:

$$
r=d \lambda \text {. }
$$

Posons, par décomposition selon les types:

$$
\lambda=\lambda_{(1,0)}+\lambda_{(0,1)}
$$

oú, d'après la réalité de $\lambda$, on a: $\bar{\lambda}_{(1,0)}=\lambda_{(0,1)}$. Pour $d \lambda$, on obtient:

$$
d \lambda=d^{\prime} \lambda_{(1,0)}+\left(d^{\prime \prime} \lambda_{(1,0)}+d^{\prime} \lambda_{(0,1)}\right)+d^{\prime \prime} \lambda_{(0,1)} .
$$

Pour que $\gamma=d \lambda$ soit de type $(1,1)$, il faut et il suffit que $d^{\prime} \lambda_{(1,0)}=0 ; \lambda_{(1,0)}$ étant $d^{\prime}$-fermée peut s'écrire:

$$
\lambda_{(1,0)}=d^{\prime} \rho+h
$$

où $h$ est une 1-forme holomorphe $\left(d^{\prime \prime} h=0\right)$ et $\rho$ un scalaire complexe. On en déduit:

$$
d \lambda=d^{\prime \prime} d^{\prime} \rho+d^{\prime} d^{\prime \prime} \bar{\rho}=d^{\prime} d^{\prime \prime}(\bar{\rho}-\rho) .
$$

Posons $\bar{\rho}-\rho=i \varphi$, où $\varphi$ est un scalaire réel. Il résulte de (5.1) que si $\gamma$ de type $(1,1)$ est homologue à 0 , il existe un scalaire réel $\varphi$ tel que:

$$
\gamma=i d^{\prime} d^{\prime \prime} \varphi
$$

Nous introduisons dans la suite le scalaire positif $f=\exp \varphi$ et écrivons (5.2) sous la forme:

$$
\gamma=i d^{\prime} d^{\prime \prime} \log f \quad(f>0) .
$$

Inversement à tout scalaire strictement positif $f,(5.3)$ fait correspondre une 2 -forme réelle $\gamma$ de type $(1,1)$ homologue à 0 . Si $c$ est la forme hermitienne associée à $\gamma$, on a en coordonnées locales:

$$
c_{\alpha \beta}=\partial_{\alpha} \partial_{\beta} \log f \quad(f>0)
$$

ou indifféremment :

$$
c_{\alpha \beta}=\nabla_{\alpha} \nabla_{\beta} \log f \text {. }
$$

b) Supposons que $c_{1}\left(W_{n}\right)=0$. De l'étude précédente, il résulte que pour qu'il en soit ainsi, il faut et il suffit qu'il existe un scalaire positif $f$ tel que:

$$
R_{\alpha \beta}=\partial_{\alpha} \partial_{\beta} \log f \text {. }
$$

Nous sommes ainsi conduits à attacher à chaque domaine $U$ de coordonnées 
locales complexes le scalaire local strictement positif $k_{U}=f \sqrt{g_{U}}$ qui d'après (4.4) et (5.5) vérifie :

$$
\partial_{\alpha} \partial_{\beta} \log k_{U}=\partial_{\alpha} \partial_{\beta} \log f+\partial_{\alpha} \partial_{\beta} \log \sqrt{g_{U}}=0
$$

De (5.6) on déduit immédiatement qu'il existe une function holomorphe locale $a_{v}$ telle que:

$$
k_{U}=a_{U}(z) \overline{a_{U}(z)}
$$

$k_{U}$ étant strictement positif, $a_{U}$ ne saurait s'annuler sur $U$.

Les $k_{U}$ correspondant aux différents domaines de coordonnées locales définissent sur $W_{n}$ la densité scalaire (ou noyau) $k$ associée à la 2 n-forme $f_{\eta}$. Soit $V$ un domaine tel que $U \cap V$ soit connexe, $\mathscr{J}_{V}^{U}(z)$ le jacobien correspondant à $U$ et $V$. Si $k_{V}$ est la composante de $k$ relative au domaine $V$, on a d'après la définition des densités scalaires, sur $U \cap V$,

$$
k_{V}=\mathscr{J}_{V}^{U} \bar{J}_{V}^{U} k_{U} .
$$

Il vient ainsi :

$$
a_{V}(z) \overline{a_{V}(z)}=\mathscr{J}_{V}^{U}(z) \overline{\mathscr{J}_{V}^{U}(z)} a_{U}(z) \overline{a_{U}(z)} \quad(z \in U \cap V) .
$$

Il en résulte que les quantités

$$
C_{V}^{U}=\frac{a_{V}(z)}{\mathscr{J}_{V}^{U}(z) a_{U}(z)}=\frac{\overline{\mathscr{J}_{V}^{U}(z)} \overline{a_{U}(z)}}{\overline{a_{V}(z)}}
$$

sont sur $U \cap V$ des constantes $\neq 0$. On a donc:

$$
a_{V}(z)=C_{V}^{U} \mathscr{J}_{V}^{U}(z) a_{U}(z)
$$

avec $C_{V}^{U} \overline{C_{V}^{U}}=1$. Il existe donc des constantes réelles $\varphi_{V}^{U}$ telles que:

$$
a_{V}(z)=e^{i_{\varphi}^{U}} \mathscr{J}_{V}^{U}(z) a_{U}(z) \quad(z \in U \cap V) .
$$

Soit $W$ un troisième domaine de coordonnées locales tel que $U \cap W$ et $V \cap W$ soient connexes. On a

$$
a_{W}(z)=e^{i \varphi} \mathscr{J}_{W}^{V}(z) a_{V}(z) \quad(z \in V \cap W) .
$$

Si $z \in U \cap V \cap W$, on a donc:

$$
a_{W}(z)=e^{i\left(\varphi_{V}^{U}+\varphi_{W}^{V}\right)} \mathscr{J}_{V}^{U}(z) \mathscr{J}_{W}^{V}(z) a_{U}(z)
$$


Il en résulte que les constantes introduites vérifient:

$$
\varphi_{W}^{U} \equiv \varphi_{V}^{U}+\varphi_{W}^{V} \quad(\bmod .2 \pi) .
$$

c) Soit $\left(\tilde{W}_{n}, p\right)$ le revêtement universel de $W_{n}$. Nous munissons $\tilde{W}_{n}$ des éléments images réciproques par $p$ de ceux introduits sur $W_{n}$. Introduisons un recouvrement de $\tilde{W}_{n}$ par des domaines $\bar{U}, \bar{V}, \tilde{W}$, etc.... tels que l'intersection de deux domaines soit connexe. Soit $\varphi_{\tilde{U}}$ une constante réelle attachée au domaine $\bar{U}$. On en déduit pour un domaine $\bar{V}$ tel que $\bar{U} \cap \bar{V} \neq \phi$ une constante $\varphi_{\tilde{V}}$ définie par:

$$
\varphi_{\widetilde{V}}^{\widetilde{U}} \equiv \varphi_{\tilde{U}}-\varphi_{\tilde{V}} \quad(\bmod .2 \pi)
$$

Si $\bar{U} \cap \tilde{V} \cap \tilde{W} \neq \phi$, il $y$ a cohérence d'après l'analogue de (5.9), puisque :

$$
\varphi_{\widetilde{W}}^{\tilde{V}} \equiv \varphi_{\widetilde{W}}^{\tilde{U}}-\varphi_{\widetilde{V}}^{\tilde{U}} \equiv\left(\varphi_{\tilde{U}}-\varphi_{\tilde{W}}\right)-\left(\varphi_{\tilde{U}}-\varphi_{\tilde{V}}\right) \equiv \varphi_{\tilde{V}}-\varphi_{\tilde{W}} . \quad(\bmod .2 \pi) .
$$

Ces constantes modulo $2 \pi$ étant ainsi choisies pour le recouvrement envisagé de $\tilde{W}_{n}$, on a

$$
e^{i \varphi} \tilde{v} \tilde{a}_{\tilde{v}}(\tilde{z})=\mathscr{J}_{\tilde{V}}^{\tilde{U}}(\tilde{z}) e^{i \varphi} \tilde{U} \tilde{a}_{\tilde{U}}(\tilde{z})
$$

(5.10) exprime que les quantités $e^{i \varphi} \tilde{U} \tilde{a}_{\tilde{U}}(\bar{z})$ sont les composantes sur $\tilde{W}_{n}$ d'une $n$-forme holomorphe $\tilde{\alpha}$, partout différente de zéro. Si $s$ est un actomorphisme de $\tilde{W}_{n}$ correspondent à un élément du groupe fondamental $\pi_{1}\left(W_{n}\right)$, on a

$$
s^{*} \tilde{\alpha}=e^{i \varphi(s)} \tilde{\alpha}
$$

où $\varphi(s)$ est une constante réelle, définie modulo $2 \pi$.

Anisi si $c_{1}\left(W_{n}\right)=0$, il existe sur $\tilde{W}_{n}$ une $n$-forme holomorphe $\tilde{\alpha}$ partout $\neq 0$, vérifiant $(5.11)$.

Un recouvrement satisfaisant à la condition énoncée peut, par exemple, étre construit au moyen de "voisinages convexes" (voir Kobayashi-Nomizu ([5], p 166) pour la structure riemannienne envisagée.

d) Inversement supposant qu'il existe sur $\bar{W}_{n}$ une $n$-forme holomorphe $\tilde{\alpha}$ partout $\neq 0$, vérifiant (5.11), pour chaque élément de $\pi_{1}\left(W_{n}\right)$. La 2 -forme $\bar{K}=\tilde{\alpha} \wedge \overline{\tilde{\alpha}}$, partout $\neq 0$, de $\tilde{W}_{n}$ satisfait

$$
s^{*} \bar{K}=\bar{K} \text {. }
$$

Cette forme définit donc une 2 n-forme de $W_{n}$ dont le noyan $k$ qui peut s'écrire localement $k=\tilde{a} \overline{\tilde{a}}$ vérifie:

$$
-(2 \pi)^{-1} i \partial_{\alpha} \partial_{\beta} \log k_{U}=0 .
$$


Le premier membre définit une 2-forme de la classe de Chern. Il en résulte que $c_{1}\left(W_{n}\right)=0$. Nous pouvons énoncer

Théorèm. Pour que la première classe de Chern $c_{1}\left(W_{n}\right)$ d'une variété kählerienne compacte $W_{n}$ soit nulle, il faut et il suffit qu'il existe sur le revêtement universel $\tilde{W}_{n}$ une $n$-forme holomorphe $\tilde{\alpha}$ partout différente de 0 et vérifiant (5.11) pour tout élément de $\pi_{1}\left(W_{n}\right)$.

\section{Sous-algèbre de $L_{h}$ laissant invariante une $2 n$-forme $\geqslant 0$}

Donnons-nous sur $W_{n}$, kählerienne compacte, une 2 n-forme non nulle

$$
K=f_{\eta} \quad(f \geqslant 0)
$$

où $f$ est un scalaire $\geqslant 0$. Par abus de langage, nous dirons que la $2 n$-forme $K$ est supposée $\geqslant 0$.

A $K$ est canoniquement associée un noyau $k$ dont la composante relative à un domaine $U$ est:

$$
k_{U}=f \sqrt{g_{U}} \geqslant 0 .
$$

a) Soit $L_{h}$ l'algèbre complexe de toutes les t-i holomorphes de $W_{n}, L$ une sous algèbre complexe de $L_{h}$. Nous nous proposons d'exprimer que $L$ laisse invariante la forme $K$ ou-ce qui est équivalent-le noyau $k$ [11].

La relation $\mathscr{L}(X) k=0$ x'exprime localement, sur un domaine $U$, par:

$$
\left(X^{\alpha} \partial_{\alpha} k_{U}+\partial_{\alpha} X^{\alpha} k_{U}\right)+\left(X^{\star} \partial_{\alpha} k_{U}+\partial_{\alpha} X^{\star} k_{U}\right)=0 .
$$

De meme $\mathscr{L}(J X) k=0$ s'écrit :

$$
i\left(X^{\alpha} \partial_{\alpha} k_{U}+\partial_{\alpha} X^{\alpha} k_{U}\right)-i\left(X^{a} \partial_{\alpha} k_{U}+\partial_{\alpha} X^{a} k_{U}\right)=0 .
$$

Ainsi pour que $X$ et $J X$ laissent $k$ invariant, il faut et il suffit que:

$$
X^{\alpha} \partial_{\alpha} k_{U}+\partial_{\alpha} X^{\alpha} k_{U}=0 \text {. }
$$

D'après (6.2) la relation précédente peut s'écrire:

$$
X^{\alpha}\left(\partial_{\alpha} f \sqrt{g_{U}}+f \partial_{\alpha} \sqrt{g_{U}}\right)+\partial_{\alpha} X^{\alpha} f \sqrt{g_{U}}=0
$$

soit :

$$
f\left(\partial_{\alpha} X^{\alpha}+\frac{\partial_{\lambda} \sqrt{g_{U}}}{\sqrt{g_{U}}} X^{\lambda}\right)+X^{\alpha} \partial_{\alpha} f=0
$$

Or, d'après des formules classiques de géométrie kählérienne [8]: 


$$
\nabla_{\alpha} X^{\alpha}=\partial_{\alpha} X^{\alpha}+\Gamma_{\alpha \lambda}^{\alpha} X^{\lambda}=\partial_{\alpha} X^{\alpha}+\frac{\partial_{2} \sqrt{g_{U}}}{\sqrt{g_{U}}} X^{\lambda}
$$

(6.4) peut ainsi s'écrire

$$
f \nabla_{\alpha} X^{\alpha}+X^{\alpha} \nabla_{\alpha} f=0
$$

c'est-à-dire

$$
\nabla_{\alpha}\left(f X^{\alpha}\right)=0
$$

Si $\xi=\alpha\left(X^{(1,0)}\right)$ est la 1 -forme de type $(1,0)$ associée à $X^{(1,0)}$, la relation $(6.5)$ est équivalente à

$$
\delta^{\prime}(f \xi)=0
$$

Ainsi pour que $L$ laisse $K$ invariante, il faut et il suffit que la 1-forme $\xi=\alpha\left(X^{(1,0)}\right)$ associée à tout élément $X$ de $L$ vérifie la relation (6.6).

b) Si $X \in L$, la 1 -forme $d^{\prime}$-fermée $\xi$ peut s'écrire:

$$
\xi=d^{\prime} \rho+H \xi \text {. }
$$

Si $X \in L \cap I, \xi$ est $d^{\prime}$-homologue à 0 :

$$
\xi=d^{\prime} \rho .
$$

$\xi$ vérifiant (6.6), on a :

$$
\left\langle f d^{\prime} \rho, d^{\prime} \rho\right\rangle=\left\langle\delta^{\prime}\left(f d^{\prime} \rho\right), \rho\right\rangle=0
$$

et par suite, $f$ étant $\geqslant 0, f d^{\prime} \rho=0$. Soit $V$ un domaine de $W_{n}$ sur lequel $f$ ne s'annule pas; $d^{\prime} \rho=\xi$, donc le champ de vecteurs $X$ correspondant, sont identiquement nuls sur $V$. Par analyticité, $X$ est identiquement nul sur $W_{n}$ et $L \cap I=0$.

En particulier $[L, L]$ qui est contenu dans $L \cap I$ est nul.

Considérons l'application linéaire de l'espace vectoriel complexe $L$ dans l'espace vectoriel complexe $H^{(1)}$ des 1 -formes holomorphes définie par:

$$
X \in L \rightarrow H \xi=H \alpha\left(X^{(1,0)}\right) \in H^{(1)} .
$$

Cette application est injective puisque son noyau est $L \cap I=0$. Il en résulte:

$$
\operatorname{dim}_{C} L \leqslant \operatorname{dim}_{C} H^{(1)}=b_{1,0}\left(W_{n}\right) .
$$

Lemme 1. Toute sous-algèbre complexe $L$ de $L_{h}$ qui laisse invariante une $2 n$-forme $K \geqslant 0$ est abélienne et:

$$
\operatorname{dim}_{C} L \leqslant b_{1,0}\left(W_{n}\right)
$$


c) Soit $T^{p}$ l'espace complexe des $p$-tenseurs holomorphes sur $W_{n}$. La situation précédente et les travaux de Kodaira nous conduisent à introduire le sous-espace $U^{p}(f)$ de $T^{p}$ défini par les $p$-tenseurs holomorphes $A$ vérifiant la condition:

$$
\delta^{\prime}\{f \alpha(A)\}=0
$$

où $f$ est un scalaire $\geqslant 0([6])$.

Si $A \in U^{p}(f)$ et si $k(A)=0$, on sait que $H \alpha(A)=0$ et on déduit de (6.11) par un raisonnement identique à celui du lemme 1 que $A=0$. L'application linéaire de l'espace vectoriel complexe $U^{p}(f)$ dans l'espace vectoriel complexe $H^{(p)}$ des $p$-formes holomorphes définie par:

$$
A \in U^{p}(f) \rightarrow H \alpha(A) \in H^{(p)}
$$

est injective. Ainsi :

Lemme 2. Pour tout $f \geqslant 0$, tout élément $A$ de $U^{p}(f)$ qui vérifie $k(A)=0$ est identiquement nul et

$$
\operatorname{dim}_{C} U^{p}(f) \leqslant b_{p, 0}\left(W_{n}\right)
$$

On notera qu'il résulte de ce lemme qu'un élément $A$ de $U^{p}(f)$ ne peut $s$ 'annuler sans être identiquement nul. En effet si $A$ s'annule en un point de $W_{n}, k(A)=0$.

\section{Invariance par $L$ des éléments de $U^{p}(f)$}

a) Soit $X$ une t. i. holomorphe et soit $A$ un $p$-tenseur holomorphe. Le transformé infinitésimal de $A$ par $X$ est le tenseur de type $(p, 0)$ défini, sur un domaine $U$ de coordonnées locales complexes, par:

$$
\{\mathscr{L}(X) A\}^{\rho_{1} \cdots \rho_{p}}=X^{\lambda} \partial_{2} A^{\rho_{1} \cdots \rho_{p}}-\sum_{i=1}^{p} \partial_{2} X^{\rho_{i}} A^{\rho_{1} \cdots \lambda \cdots \rho_{p}}
$$

Les composantes de $X$ et de $A$ étant des fonctions holomorphes sur $U$, il en est de même des composantes du $p$-tenseur $\mathscr{L}(X) A$. Ainsi si $A \in T^{p}, X \in L_{h}$ on a $\mathscr{L}(X) A \in T^{p}$.

En introduisant des dérivées covariantes, (7.1) peut s'écrire:

$$
\{\mathscr{L}(X) A\}^{\rho_{1} \cdots \rho_{p}}=X^{2} \nabla_{\lambda} A^{\rho_{1} \cdots \rho_{p}}-\sum_{i=1}^{p} \nabla_{\lambda} X^{\rho_{i}} A^{\rho_{1} \cdots \lambda \cdots \rho_{p}}
$$

ou comme on le vérifie immédiatement: 


$$
\{\mathscr{L}(X) A\}^{\rho_{1} \cdots \rho_{p}}=X^{\lambda} \nabla_{2} A^{\rho_{1} \cdots \rho_{p}}-\frac{1}{(p-1) !} \varepsilon_{{ }_{0 \tau_{2}} \cdots \tau_{p}}^{\rho_{1} \cdots \rho_{p}} \nabla_{\lambda} X^{o} A^{\lambda \tau_{2} \cdots \tau_{p}}
$$

où $\varepsilon$ est le tenseur-indicateur de Kronecker.

b) Supposons maintenant que $A \in U^{p}(f)$, c'est-à-dire vérifie en outre:

$$
\delta^{\prime}\{f \alpha(A)\}=0 .
$$

Cette relation peut s'écrire sur le domaine $U$ :

$$
\nabla_{\lambda}\left(f A^{\lambda \rho_{2} \cdots \rho_{p}}\right)=0 .
$$

En explicitant la dérivation covariante, il vient sur $U$ :

$$
\nabla_{\lambda}\left(f A^{\lambda \rho_{2} \cdots \rho_{p}}\right)=\partial_{\lambda}\left(f A^{\lambda \rho_{2} \cdots \rho_{p}}\right)+\Gamma_{\lambda \rho}^{\lambda} A^{\alpha \rho_{2} \cdots \rho_{p}}=\partial_{\lambda}\left(f A^{\lambda \rho_{2} \cdots \rho_{p}}\right)+\frac{\partial_{\lambda} \sqrt{g_{U}}}{\sqrt{g_{U}}} A^{\lambda \rho_{2} \cdots \rho_{p}} .
$$

Il en résulte, par produit par $\sqrt{g_{V}}$, que (7.3) peut s'écrire:

$$
\partial_{\lambda}\left(A^{2 \rho_{2} \cdots \rho_{p}} \sqrt{g_{U}}\right)=0
$$

ou si l'on introduit le noyau $k$ de composantes $k_{U}=f \sqrt{ } \overline{g_{U}}$ :

$$
\partial_{\lambda}\left(A^{2 \rho_{2} \cdots \rho_{p}} k_{U}\right)=0 .
$$

Soit $X \in L$ laissant $K=f_{\eta}$ invariante; $\mathscr{L}(X)$ commute avec $\partial_{2}$ et annule $k$; par produit de (7.4) par $\mathscr{L}(X)$ on a ainsi :

$$
\partial_{2}\left\{(\mathscr{L}(X) A)^{\lambda \rho_{\rho} \cdots \rho_{p}} k_{U}\right\}=0 .
$$

qui exprime que $\mathscr{L}(X) A$ appartient à $U^{p}(f)$.

c) Soit $\beta$ une $p$-forme holomorphe sur $W_{n}$. Nous nous proposons d'abord d'obtenir une expression commode pour:

$$
(\alpha(\mathscr{L}(X) A), \beta)=\frac{1}{p !}\{\mathscr{L}(X) A\}^{\rho_{1} \cdots \rho_{p}} \beta_{\rho_{1} \cdots \rho_{p}} .
$$

D'après (7.2), il vient:

$$
\begin{gathered}
(\alpha(\mathscr{L}(X) A), \beta)=\frac{1}{p !} X^{2} \nabla_{\lambda}\left(A^{\rho_{1} \cdots \rho_{p}} \beta_{\rho_{1} \cdots \rho_{p}}\right)-\frac{1}{p !} X^{2} A^{\rho_{1} \cdots \rho_{p}} \nabla_{\lambda} \beta_{\rho_{1} \cdots \rho_{p}} \\
-\frac{1}{(p-1) !} \nabla_{\lambda} X^{o} A^{2 \tau_{2} \cdots \tau_{p}} \beta_{o \tau_{2} \cdots \tau_{p}} .
\end{gathered}
$$

Le premier terme du second membre étant nul, on peut écrire: 


$$
\begin{aligned}
(\alpha(\mathscr{L}(X) A), \beta)= & -\frac{1}{(p-1) !} A^{\alpha \tau_{2} \cdots \tau_{p}} \nabla_{\lambda}\left(X^{\circ} \beta_{\sigma \tau_{2} \cdots \tau_{p}}\right) \\
& +\frac{1}{(p-1) !} A^{\alpha \tau_{2} \cdots \tau_{p}} X^{\circ} \nabla_{\lambda} \beta_{o \tau_{2} \cdots \tau_{p}}-\frac{1}{p !} X^{2} A^{\rho_{1} \cdots \rho_{p}} \nabla_{2} \beta_{\rho_{1} \cdots \rho_{p}} .
\end{aligned}
$$

Soit en modifiant le nom des indices:

$$
\begin{aligned}
(\alpha(\mathscr{L}(X) A), \beta)= & -\frac{1}{(p-1) !} A^{\lambda \tau_{\tau_{2} \cdots \tau_{p}}} \nabla_{\lambda}\left(X^{o} \beta_{\sigma \tau_{2} \cdots \tau_{p}}\right) \\
& +\frac{1}{p !} X^{\lambda}\left[p A^{\sigma \tau_{2} \cdots \tau_{p}} \nabla_{o} \beta_{\lambda=2 \cdots \tau_{p}}-A^{\rho_{1} \cdots \rho_{p}} \nabla_{\lambda} \beta_{\rho_{1} \cdots \rho_{p}}\right]
\end{aligned}
$$

La forme holomorphe $\beta$ étant $d^{\prime}$-fermée, on a:

$$
\frac{1}{p !} \varepsilon_{\sigma_{0} \cdots \rho_{p}}^{\lambda \rho_{1} \cdots \rho_{p}} \nabla_{\lambda} \beta_{\rho_{1} \ldots \rho_{p}}=0
$$

En effectuant le produit contracté par $A^{\sigma_{1} \cdots \sigma_{p}}$ et distinguant les termes correspondant à $\lambda=\sigma_{0}$ ou $\lambda=\sigma_{1}, \cdots, \sigma_{p}$, il vient:

$$
A^{\sigma_{1} \cdots \sigma_{p}}\left(\nabla_{\sigma_{0}} \beta_{\sigma_{1} \cdots \sigma_{p}}-p \nabla_{\sigma_{1}} \beta_{\sigma_{0} \sigma_{2} \cdots \sigma_{p}}\right)=0 .
$$

ce qui peut s'écrire:

$$
A^{\sigma_{1} \cdots \sigma_{p}} \nabla_{\sigma_{0}} \beta_{\sigma_{1} \cdots \sigma_{p}}-p A^{\sigma_{2} \cdots \tau_{p}} \nabla_{\sigma} \beta_{\sigma_{0} \tau_{2} \cdots \tau_{p}}=0 .
$$

Le dernier terme du second membre de (7.5) est donc nul et l'on a:

$$
(\alpha(\mathscr{L}(X) A), \beta)=-\frac{1}{(p-1) !} A^{\lambda \tau_{2} \cdots \tau_{p}} \nabla_{\lambda}\left(X^{o} \beta_{\sigma \tau_{2} \cdots \tau_{p}}\right) .
$$

Par produit par $f$ il vient:

$$
(f \alpha(\mathscr{L}(X) A), \beta)=-\frac{1}{(p-1) !} f A^{\lambda \tau_{2} \cdots \tau_{p}} \nabla_{\lambda}\left(X^{\sigma} \beta_{\sigma \tau_{2} \cdots \tau_{p}}\right) .
$$

Mais $A$ appartenant à $U^{p}(f)$ vérifie (7.3). Par suite:

$$
(f \alpha \mathscr{L}(X) A, \beta)=-\frac{1}{(p-1) !} \nabla_{\lambda}\left(f A^{\lambda \varepsilon_{2} \cdots \tau_{p}} X^{\circ} \beta_{o \tau_{2} \ldots z_{p}}\right) .
$$

Par intégration sur $W_{n}$, on obtient: 


$$
\langle f \alpha(\mathscr{L}(X) A), \beta>=0 .
$$

En particulier:

$$
\langle f \alpha(\mathscr{L}(X) A), H \alpha(\mathscr{L}(X) A)\rangle=k(\mathscr{L}(X) A)<f, 1>=0 .
$$

Ainsi $k(\mathscr{L}(X) A)=0$ et du lemme 2 il résulte $\mathscr{L}(X) A=0$. Nous énonçons :

Lemme 3. Toute sous-algèbre complexe $L$ de $L_{h}$ qui laisse invariante une $2 n$-forme $K=f_{\eta} \geqslant 0$, laisse invariant chaque élément de $U^{p}(f)$.

\section{Invariance des formes holomorphes-Application}

a) Nous avons vu que sur une variété kählerienne compacte $W_{n}$, toute $p$-forme holomorphe $\beta$ est fermée. Si $X \in L_{h}$, on a:

$$
\mathscr{L}(X) \beta=(\operatorname{di}(X)+i(X) d) \beta=\operatorname{di}(X) \beta .
$$

La forme $i(X) \beta$, de type $(p-1,0)$ a pour composantes sur un domaine $U$ de coordonnées locales

$$
\{i(X) \beta\}_{\tau_{2} \cdots \tau_{p}}=X^{\lambda} \beta_{\lambda \tau_{2} \cdots \tau_{p}} .
$$

Ainsi $i(X) \beta$ est holomorphe, donc fermée et $\mathscr{L}(X) \beta=0$.

Proposition 1. Sur une variété kählerienne compacte $W_{n}$, toute p-forme holomorphe est invariante par le plus grand groupe connexe de transformations holomorphes de $W_{n}$.

b) Reprenons une variété kählerienne compacte $W_{n}$ telle que $b_{n, 0}\left(W_{n}\right) \neq 0$. Il existe donc sur $W_{n}$ une $n$-forme holomorphe $\beta$ non identiquement nulle. Celle-ci est invariante pour toute t. i. holomorphe. Il en est donc de même pour la 2 n-forme $K=\varepsilon_{n} \beta \wedge \bar{\beta}$ manifestement $\geqslant 0$. Nous posons:

$$
K=\varepsilon_{n} \beta \wedge \bar{\beta}=f_{\eta} \quad(f \geqslant 0) .
$$

Relativement à $K$, l'algèbre de Lie $L_{h}$ de toutes les t. i. holomorphes vérifie les hypothèses du lemme 1 . Ainsi:

Proposition 2. Si $W_{n}$ est une variété kählérienne compacte telle que $b_{n, 0}\left(W_{n}\right) \neq 0$, le plus grand groupe connexe $G$ de transformations holomorphes de $W_{n}$ est abélien et $\operatorname{dim}_{R} G \leqslant b_{1}\left(W_{n}\right)$.

$f$ étant défini par (8.2), il résulte du lemme 3 que le groupe $\mathrm{G}$ laisse invariant tout élément $A$ de $U^{p}(f)$.

\section{Identités relatives aux tenseurs holomorphes}

a) A tout $p$-forme $\alpha$ de type $(p, 0)$, associons le $(p+1)$-tenseur covariant de type $(p+1,0)$ défini par. 


$$
a(\alpha)_{\lambda \rho_{1} \cdots \rho_{p}}=\nabla_{\lambda} \alpha_{\rho_{1} \ldots \rho_{p}} .
$$

D'après (3.7), pour que le $p$-tenseur $A$ de type $(p, 0)$ soit holomorphe, il faut et il suffit que $a\{\alpha(A)\}=0$. Compte-tenu de la compacité de $W_{n}$, nous allons mettre cette condition du premier ordre sous la forme d'une condition du second ordre.

Etant donné un tenseur covariant $t$ de type $(1,1)$, nous introduisons l'opérateur $Q(t)$ sur les formes $\alpha$ de type $(p, 0)$ défini par:

$$
Q(t) \alpha=\frac{2}{(p-1) !} t_{2}^{\mu} \alpha_{\mu \sigma_{2} \cdots \sigma_{p}} d z^{2} \wedge d z^{\sigma_{2}} \wedge \cdots \wedge d z^{\sigma_{p}} .
$$

On en déduit sur $U$ :

$$
\{Q(t) \alpha\}_{\rho_{1} \cdots \rho_{p}}=\frac{2}{(p-1) !} \varepsilon_{\rho_{1} \cdots \rho_{p}}^{\lambda \sigma_{2} \cdots \sigma_{p}} t_{{ }^{\mu}} \alpha_{\mu \sigma_{2} \cdots \sigma_{p}} .
$$

Soit $R$ le tenseur de Ricci de la variété. D'après l'expression générale du laplacien $\Delta \alpha$ d'une forme, on a avec la notation (9.2):

$$
(\Delta \alpha)_{\rho_{1} \cdots \rho_{p}}=-\nabla^{k} \nabla_{k} \alpha_{\rho_{1} \cdots \rho_{p}}+\frac{1}{2}(Q(R) \alpha)_{\rho_{1} \cdots \rho_{p}} .
$$

On en déduit:

$$
\left(\Delta \alpha-\frac{1}{2} Q(R) \alpha\right)_{\rho_{1} \ldots \rho_{p}}=-\nabla^{2} \nabla_{2} \alpha_{\rho_{1} \ldots \rho_{p}}-\nabla_{\lambda} \nabla^{2} \alpha_{\rho_{1} \ldots \rho_{p}} .
$$

Or d'après l'identité de Ricci

$$
\left(\nabla_{\lambda} \nabla^{2}-\nabla^{2} \nabla_{2}\right) \alpha_{\rho_{1} \cdots \rho_{p}}=-\sum_{i=1}^{p} R_{\rho_{i}}{ }^{2} \alpha_{\rho_{1} \cdots 2 \ldots \rho_{p}}=-\frac{1}{(p-1) !} \varepsilon_{\rho_{1} \cdots \rho_{p}}^{2 \sigma_{2} \cdots \sigma_{p}} R_{\lambda}{ }^{\mu} \alpha_{\mu \sigma_{2} \cdots \sigma_{p}}
$$

soit:

$$
\nabla_{2} \nabla^{2} \alpha_{\rho_{1} \cdots \rho_{p}}=\nabla^{2} \nabla_{2} \alpha_{\rho_{1} \cdots \rho_{p}}-\frac{1}{2}[Q(R) \alpha]_{\rho_{1} \cdots \rho_{p}} .
$$

De (9.3) et (9.4), il résulte:

$$
(\Delta \alpha-Q(R) \alpha)_{\rho_{1} \cdots \rho_{p}}=-2 \nabla^{\lambda} \nabla_{\lambda} \alpha_{\rho_{1} \cdots \rho_{p}}
$$

c'est-à-dire l'identité:

$$
(\Delta \alpha-Q(R) \alpha)_{\rho_{1} \ldots \rho_{p}}=-2 \nabla^{2} a(\alpha)_{\lambda \rho_{1} \ldots \rho_{p}} .
$$

Si $A$ est un $p$-tenseur holomorphe $a\{\alpha(A)\}=0$ et $\alpha(A)$ est solution de l'équation elliptique: 


$$
\Delta \alpha(A)-Q(R) \alpha(A)=0 .
$$

b) Associons à un $p$-tenseur $A$ de type $(p, 0)$ la 1-forme $b(A)$ définie par:

$$
b(A)_{2}=\frac{1}{p !} A^{\rho_{1} \cdots \rho_{p}} a\{\alpha(A)\}_{\lambda \rho_{1} \cdots \rho_{p}} .
$$

Dans la suite, nous écrivons $a(\alpha)$ pour $a\{\alpha(A)\}$. Par dérivation il vient:

$$
\delta^{\prime} b(A)=-\frac{1}{p !} \nabla^{2} A^{\rho_{1} \cdots \rho_{p}} a(\alpha)_{\lambda \rho_{1} \cdots \rho_{p}}-\frac{1}{p !} A^{\rho_{1} \cdots \rho_{p}} \nabla^{2} a(\alpha)_{\lambda \rho_{1} \cdots \rho_{p}} .
$$

De (9.5) on déduit ainsi:

$$
(\alpha, \Delta \alpha-Q(R) \alpha)=2(p+1)(a(\alpha), a(\alpha))+2 \delta^{\prime} b(A) .
$$

Par intégration sur $W_{n}$, il vient:

$$
\langle\Delta \alpha-Q(R) \alpha, \alpha>=2(p+1)<a(\alpha), a(\alpha)\rangle
$$

où le second membre est positif et n'est nul que pour $a(\alpha)=0$. Ainsi (voir [8]):

Proposition 1. Etant donnée une variété kählerienne compacte $W_{n}$, on a pour toute forme $\alpha$ de type $(p, 0)$ la relation:

$$
\langle\Delta \alpha-Q(R) \alpha, \alpha>=2(p+1)\langle a(\alpha), a(\alpha)\rangle .
$$

Pour qu'un p-tenseur A de type $(p, 0)$ soit holomorphe, il faut et il suffit que $\alpha(A)$ vérifie:

$$
<\Delta \alpha(A)-Q(R) \alpha(A), \alpha(A)>=0
$$

ou soit solution de l'équation:

$$
\Delta \alpha(A)-Q(R) \alpha(A)=0 .
$$

\section{Introduction d'un scalaire $f>0$}

a) Sur la vaiété kählerienne compacte $W_{n}$, donnons-nous un scalaire $f>0$ et introduisons, en accord avec les travaux de Kodaira, l'espace complexe $F^{p}(f)$ des $p$-formes $\alpha$ de type $(p, 0), d^{\prime}$-fermées

$$
d^{\prime} \alpha=0
$$

et vérifiant la condition

$$
\delta^{\prime}(f \alpha)=0
$$


Soit $\mathscr{F}$ le fibré vectoriel complexe trivial de $W_{n}, \Omega^{\circ}(\mathscr{F})$ le faisceau des fonctions holomorphes à coefficients dans $\mathscr{F}$. D'après Kodaira:

$$
\operatorname{dim}_{C} F^{p}(f)=\operatorname{dim}_{C} H^{p}\left(W_{n}, \Omega^{\circ}(\mathscr{F})\right)
$$

et d'après un résultat classique de Dolbeault:

$$
\operatorname{dim}_{C} H^{p}\left(W_{n}, \Omega^{\circ}(\mathscr{F})\right)=b_{p, \text { C }}\left(W_{n}\right) .
$$

Il vient ainsi :

$$
\operatorname{dim}_{C} F^{p}(f)=b_{p, 0}\left(W_{n}\right)
$$

Introduisons sur les formes $\alpha$ de type $(p, 0)$ les opérateurs :

$$
\delta_{f}^{\prime} \alpha=\bar{f}^{1} \delta^{\prime}(f \alpha), \quad \Delta_{f}=2\left(d^{\prime} \delta_{f}^{\prime}+\delta_{f}^{\prime} d^{\prime}\right)
$$

et le produit scalaire global:

$$
\langle\alpha, \beta\rangle_{f}=\langle\alpha, f \beta\rangle \text {. }
$$

$\delta_{f}^{\prime}$ est le transposé de $d^{\prime}$ pour ce produit scalaire. En effet si $\alpha$ est une forme de type $(p+1,0)$ et $\beta$ une forme de type $(p, 0)$, on a:

$$
\left\langle\delta_{f}^{\prime} \alpha, \beta\right\rangle_{f}=\left\langle\delta^{\prime}(f \alpha), \beta\right\rangle=\left\langle f \alpha, d^{\prime} \beta\right\rangle=\left\langle\alpha, d^{\prime} \beta\right\rangle_{f} .
$$

$\Delta_{f}$ est une généralisation du laplacien $\Delta$ de G. de Rham. Si $\alpha$ est une forme de type $(p, 0)$ :

$$
\left\langle\Delta_{f} \alpha, f \alpha\right\rangle=\left\langle\Delta_{f} \alpha, \alpha\right\rangle_{f}=2\left\langle\delta_{f}^{\prime} \alpha, \delta_{f}^{\prime} \alpha\right\rangle_{f}+2\left\langle d^{\prime} \alpha, d^{\prime} \alpha\right\rangle_{f} .
$$

Il vient ainsi:

$$
\left\langle\Delta_{f} \alpha, f \alpha>=2\left\langle\delta_{f}^{\prime} \alpha, f \delta_{f}^{\prime} \alpha>+2\left\langle d^{\prime} \alpha, f d^{\prime} \alpha\right\rangle\right.\right.
$$

Nous voyons que $\left\langle\Delta_{f} \alpha, f \alpha\right\rangle$ est positif et n'est nul que pour:

$$
d^{\prime} \alpha=0, \quad \delta_{f}^{\prime} \alpha=0
$$

c'est-à-dire pour les éléments de $F^{p}(f)$. Il en résulte que les éléments de $F^{p}(f)$ coincident avec les solutions de l'équation:

$$
\Delta_{f} \alpha=0 \text {. }
$$

b) Il est aisé de donner de $\Delta_{f}$ une expression simple en fonction de $\Delta$ et de $f$. Pour une forme $\alpha$ de type $(p, 0)$, on a:

$$
\Delta_{f} \alpha=2 d^{\prime}\left\{f^{-1} \delta^{\prime}(f \alpha)\right\}+2 f^{-1} \delta^{\prime}\left(f d^{\prime} \alpha\right) .
$$


On en déduit en explicitant en coordonnées locales les dérivations:

$$
\left(\Delta_{f} \alpha\right)_{\rho_{1} \ldots \rho_{p}}=-\frac{2}{(p-1) !} \varepsilon_{\rho_{1} \cdots \rho_{p}}^{\mu \sigma_{2} \cdots \sigma_{p}} \nabla_{\mu}\left\{f^{-1} \nabla^{\lambda}\left(f \alpha_{\lambda \sigma_{2} \cdots \rho_{p}}\right)\right\}-2 f^{-1} \nabla^{2}\left\{f\left(d^{\prime} \alpha\right)_{\lambda \rho_{1} \ldots \rho_{p}}\right\}
$$

ou en développant:

$$
\begin{aligned}
& \left(\Delta_{f} \alpha\right)_{\rho_{1} \cdots \rho_{p}}=-\frac{2}{(p-1) !} \varepsilon_{\rho_{1} \cdots \rho_{p}}^{\mu \sigma_{2} \cdots \sigma_{p}} \nabla_{\mu} \nabla^{\lambda} \alpha_{2 \sigma_{2} \cdots \sigma_{p}}-2 \nabla^{\lambda}\left(d^{\prime} \alpha\right)_{2 \rho_{1} \cdots \rho_{p}} \\
& -\frac{2}{(p-1) !} \varepsilon_{\rho_{1} \cdots \rho_{p}}^{\mu \sigma_{2} \cdots \sigma_{p}} \nabla_{\mu}\left\{\left(f^{-1} \nabla^{2} f\right) \alpha_{\lambda \alpha_{2} \cdots o_{p}}\right\}-2\left(f^{-1} \nabla^{2} f\right)\left(d^{\prime} \alpha\right)_{\lambda \rho_{1} \ldots \rho_{p}} .
\end{aligned}
$$

Sous forme intrinsèque, il vient:

$$
\Delta_{f}=\Delta-2 d^{\prime} i\left(f^{-1} d^{\prime \prime} f\right)-2 i\left(f^{-1} d^{\prime \prime} f\right) d^{\prime}
$$

où, pour une forme $\alpha$ de type $(p, 0)$, on a posé:

$$
\left\{i\left(f^{-1} d^{\prime \prime} f\right) \alpha\right\}_{\sigma_{2} \ldots \sigma_{p}}=\left(f^{-1} \nabla^{\lambda} f\right) \alpha_{\lambda \sigma_{2} \ldots \sigma_{p}} .
$$

c) Nous posons dans la suite:

$$
R_{\alpha \beta}=c_{\alpha \beta}+\partial_{\alpha} \partial_{\beta} \log f \quad(f>0)
$$

où la forme hermitienne $c$ définit ainsi une 2-forme réelle fermée $\gamma$ de type $(1,1)$ telle que $[\gamma]$ est proportionnelle à $c_{1}\left(W_{n}\right)$. On peut obtenir, pour $f>0$ arbitraire, une généralisation de la proposition du paragraphe précédent. D'après (9.7):

$$
(f \alpha, \Delta \alpha-Q(R) \alpha)=2(p+1)(f a(\alpha), a(\alpha))+2 f \delta^{\prime} b(A) .
$$

Or:

$$
f \delta^{\prime} b(A)=\delta^{\prime}(f b(A))+i\left(d^{\prime \prime} f\right) b(A)
$$

où

$$
i\left(d^{\prime \prime} f\right) b(A)=\nabla^{2} f A^{\rho_{1} \cdots \rho_{p}} \nabla_{\lambda} \alpha(A)_{\rho_{1} \ldots \rho_{p}}=\left(f^{-1} \nabla^{2} f\right) \nabla_{2} \alpha(A)_{\rho_{1} \ldots \rho_{p}} f A^{\rho_{1} \ldots \rho_{p}} .
$$

Posons:

$$
\left(\nabla_{f^{-1} d^{\prime \prime} f} \alpha\right)_{\rho_{1} \cdots \rho_{p}}=\left(f^{-1} \nabla^{2} f\right) \nabla_{\lambda} \alpha_{\rho_{1} \cdots \rho_{p}} .
$$

On obtient ainsi:

(10.9) $\left(f \alpha, \Delta \alpha-Q(R) \alpha-2 \nabla_{f^{-1} d^{\prime \prime} f} \alpha\right)=2(p+1)(f a(\alpha), a(\alpha))+2 \delta^{\prime}\{f b(A)\}$. 
Par intégration sur $W_{n}$, nous obtenons:

$\left.(10.10)<\Delta \alpha-Q(R) \alpha-2 \nabla_{f^{-1} d^{\prime \prime} f} \alpha, f \alpha\right\rangle=2(p+1)\langle a(\alpha), f a(\alpha)\rangle$

où le second membre est encore positif et n'est nul que pour $a(\alpha)=0$.

d) De la décomposition (10.8) de $R$ on déduit d'autre part que si $\alpha$ est une forme de type $(p, 0)$ :

$$
\{Q(R) \alpha-Q(c) \alpha\}_{\rho_{1} \cdots \rho_{p}}=\frac{2}{(p-1) !} \varepsilon_{\rho_{1} \cdots \rho_{p}}^{i \sigma_{2} \cdots \sigma_{p}} \nabla_{\lambda} \nabla^{\mu} \log f \alpha_{\mu \sigma_{2} \cdots \sigma_{p}}
$$

Il en résulte

$$
\begin{aligned}
\{Q(R) \alpha-Q(c) \alpha\}_{\rho_{1} \cdots \rho_{p}}= & \frac{2}{(p-1) !} \varepsilon_{\rho_{1} \cdots \rho_{p}}^{2 \sigma_{2} \cdots \sigma_{p}} \nabla_{2}\left\{f^{-1} \nabla^{\mu} f \alpha_{\mu \sigma_{2} \cdots \sigma_{p}}\right\} \\
& -\frac{2}{(p-1) !} \varepsilon_{\rho_{1} \cdots \rho_{p}}^{2 \sigma_{2} \cdots \sigma_{p}} f^{-1} \nabla^{\mu} f \nabla_{\lambda} \alpha_{\mu \sigma_{2} \cdots \sigma_{p}}
\end{aligned}
$$

ce qui peut s'écrire, avec la notation introduite dans (10.7):

$$
\begin{aligned}
& \left\{Q(R) \alpha-Q(c) \alpha-2 d^{\prime} i\left(f^{-1} d^{\prime \prime} f\right) \alpha\right\}_{\rho_{1} \cdots \rho_{p}} \\
& =-\frac{2}{(p-1) !} \varepsilon_{\rho_{1} \cdots \rho_{p}}^{\lambda \sigma_{2} \cdots \sigma_{p}} f^{-1} \nabla^{\mu} f \nabla_{\lambda} \alpha_{\mu \sigma_{2} \cdots p_{p}} .
\end{aligned}
$$

Considérons la forme $2 i\left(f^{-1} d^{\prime \prime} f\right) d^{\prime} \alpha$ qui a pour composantes :

$$
2\left\{i\left(f^{-1} d^{\prime \prime} f\right) d^{\prime} \alpha\right\}_{\rho_{1} \cdots \rho_{p}}=\frac{2}{p !} \varepsilon_{\rho_{0} \cdots \rho_{p}}^{2 \sigma_{1} \cdots \sigma_{p}} f^{-1} \nabla^{\rho_{0}} f \nabla_{\lambda} \alpha_{\sigma_{1} \cdots \sigma_{p}} .
$$

En distinguant le cas $\rho_{0}=\lambda$ et le cas $\rho_{0}=\sigma_{1}, \cdots, \sigma_{p}$, il vient:

$$
\begin{aligned}
2\left\{i\left(f^{-1} d^{\prime \prime} f\right) d^{\prime} \alpha\right\}_{\rho_{1} \cdots \rho_{p}}= & 2 f^{-1} \nabla^{\rho_{0} f} \nabla_{\rho_{0}} \alpha_{\rho_{1} \cdots \rho_{p}} \\
& -\frac{2}{(p-1) !} \varepsilon_{\rho_{1} \cdots \rho_{p}}^{\lambda \sigma_{2} \cdots \rho_{p}} f^{-1} \nabla^{\rho_{0}} f \nabla_{\lambda} \alpha_{\rho_{0} \sigma_{2} \cdots \sigma_{p}} .
\end{aligned}
$$

De (10.11) et (10.12), on déduit ainsi que si $\alpha$ est une forme de type $(p, 0)$, on a la relation:

(10.13) $Q(R) \alpha+2 \nabla_{f^{-1} d_{f} f} \alpha=Q(c) \alpha+2\left\{d^{\prime} i\left(f^{-1} d^{\prime \prime} f\right)+i\left(f^{-1} d^{\prime \prime} f\right) d^{\prime}\right\} \alpha$ et (10.10) peut s'écrire compte-tenu de l'expression (10.6) de $\Delta_{f}$ :

$$
\left\langle\Delta_{f} \alpha-Q(c) \alpha, f \alpha>=2(p+1)<a(\alpha), f a(\alpha)\right\rangle .
$$


Nous pouvons énoncer:

Proposition 2. Etant donnée une variété kählerienne compacte $W_{n}$ et un scalaire $f>0$ sur $W_{n}$, on a pour toute forme $\alpha$ de type $(p, 0)$ la relation:

$$
\left\langle\Delta_{f} \alpha-Q(c) \alpha, f \alpha\right\rangle=2(p+1)\langle a(\alpha), f a(\alpha)>\text {. }
$$

Pour qu'un p-tenseur $A$ de type $(p, 0)$ soit holomorphe, il faut et il suffit que $\alpha(A)$ vérifie:

$$
<\Delta_{f} \alpha(A)-Q(c) \alpha(A), f \alpha(A)>=0
$$

ou soit solution de l'équation:

$$
\Delta_{f} \alpha(A)-Q(c) \alpha(A)=0
$$

11. Variété kählerienne à classe de Chern $c_{1}\left(W_{n}\right) \leqslant 0$

Supposons que la variété kählerienne compacte $W_{n}$ admette au sens du paragraphe 4 , une première classe de Chern $c_{1}\left(W_{n}\right) \leqslant 0$. Le tenseur de Ricci de la variété vérifie alors la relation (10.8), soit:

$$
R_{\alpha \beta}=c_{\alpha \beta}+\partial_{\alpha} \partial_{\beta} \log f \quad(f>0)
$$

où la forme hermitienne $c$, de coefficients $c_{\alpha \beta}$, est négative ou nulle en tout point de $W_{n}$.

a) $\mathrm{Si} \alpha$ est une $p$-forme arbitraire de type $(p, 0)$, considérons la forme $Q(c) \alpha$ donnée par

$$
(Q(c) \alpha)_{\rho_{1} \cdots \rho_{p}}=\frac{2}{(p-1) !} \varepsilon_{\rho_{1} \cdots \rho_{p}}^{2 \sigma_{2} \cdots \sigma_{p}} c_{2}{ }^{\mu} \alpha_{\mu \sigma_{2} \cdots \sigma_{p}} .
$$

Par abus de notation, nous notons encore $\alpha$ le $p$-tenseur associé à la forme $\alpha$ par conjugaison et dualité définie par la métrique. Il vient:

$$
(Q(c) \alpha, \alpha)=\frac{2}{(p-1) !} \alpha^{\alpha \sigma_{2} \cdots \sigma_{p}} c_{\lambda}{ }^{\mu} \alpha_{\mu \sigma_{2} \cdots \sigma_{p}} .
$$

Soit

$$
(Q(c) \alpha, \alpha)=\frac{2}{(p-1) !} c_{2 \bar{\mu}} \alpha^{2 \sigma_{2} \cdots \sigma_{p}} \alpha_{\sigma_{2} \cdots \sigma_{p}}
$$

De l'hypothèse faite sur $c$, il résulte ainsi:

$$
(Q(c) \alpha, \alpha) \leqslant 0
$$

en tout point de $W_{n}$. 
b) Soit $A \in T^{p}$ un p-tenseur holomorphe arbitraire de $W_{n}$. D'après la proposition 2 du paragraphe 10

$$
<\Delta_{f} \alpha(A)-Q(c) \alpha(A), f \alpha(A)>=0 .
$$

Mais d'après (10.5) et (11.3):

$$
<\Delta_{f} \alpha(A), f \alpha(A)>\geqslant 0, \quad-<Q(c) \alpha(A), f \alpha(A)>\geqslant 0 .
$$

Il en résulte, en vertu de (10.5), $\delta_{f}^{\prime} \alpha(A)=0$, soit la relation:

$$
\delta^{\prime}\{f \alpha(A)\}=0
$$

et $T^{p}$ coïncide avec $U^{p}(f)$. Il en est en particulier ainsi pour $p=1$, c'est-àdire pour l'espace des vecteurs holomorphes. Par suite l'algèbre $L_{h}$ laisse invariante la 2 n-forme positive $K=f_{\eta}$. Des lemmes 1 et 3 , on déduit le théorème suivant:

Théorème 1. Si une variété kählerienne compacte $W_{n}$ vérifie $c_{1}\left(W_{n}\right) \leqslant 0$, tout p-tenseur holomorphe est invariant par le plus grand groupe connexe $G$ de transformations holomorphes de $W_{n}$ et:

$$
\operatorname{dim}_{C} T^{p} \leqslant b_{p, \mathrm{c}}\left(W_{n}\right) .
$$

En particulier $G$ est abélien et:

$$
\operatorname{dim}_{R} G \leqslant b_{1}\left(W_{n}\right) .
$$

12. Variété kählerienne à classe de Chern $c_{1}\left(W_{n}\right) \geqslant 0$

Supposons maintenant que la variété kählerienne compacte $W_{n}$ admette, au sens du paragraphe 4 , une première classe de Chern $c_{1}\left(W_{n}\right) \geqslant 0$. On a encore (11.1) où la forme hermitienne $c$ est positive ou nulle en tout point de $W_{n}$.

a) Si $\alpha \in F^{p}(f)$, on a $\Delta_{f} \alpha=0$ et il résulte de (10.14):

$$
\langle Q(c) \alpha, f \alpha\rangle=-2(p+1)\langle a(\alpha), f a(\alpha)\rangle .
$$

Mais d'après (11.2) et l'hypothèse faite sur $c$

$$
\langle Q(c) \alpha, f \alpha>\geqslant 0 .
$$

On en déduit:

$$
\langle a(\alpha), f a(\alpha)>=0, \quad\langle Q(c) \alpha, f \alpha\rangle=0
$$

et par suite $a(\alpha)=0$. Ainsi $\alpha \in F^{p}(f)$ est nécessairement forme associée à un $p$-tenseur holomorphe $A \in U^{p}(f)$ et l'on a: 


$$
\operatorname{dim}_{C} U^{p}(f)=\operatorname{dim}_{C} F^{p}(f)=b_{p, 0}\left(W_{n}\right)
$$

Il résulte de lemme 2 du paragraphe 6 que si la constante $(\alpha, H \alpha)$ est nulle, $\alpha$ est nécessairement nulle. L'application linéaire de l'espace vectoriel complexe $F^{p}(f)$ dans l'espace vectoriel complexe $H^{(p)}$ définie par:

$$
\alpha \in F^{p}(f) \rightarrow H \alpha \in H^{(p)}
$$

est bijective. D'autre part une $p$-forme holomorphe de $W_{n}$ ne peut s'annuler en un point sans être identiquement nulle. Il en résulte:

$$
b_{p, 0}\left(W_{n}\right) \leqslant C_{n}^{p}
$$

b) Considérons en particulier le cas $p=1$. Soit $L$ la sous-algèbre complexe de $L_{h}$ définie par les t.i. holomorphes laissant invariante la 2 n-forme positive $K=f_{\eta}$. Cette sous algébre de dimension $b_{1,0}\left(W_{n}\right)$ est abélienne d'après le lemme 1 du paragraphe 6 et laisse invariant chaque élément de $U^{p}(f)$ d'après le lemme 3.

Si $I$ est l'idéal introduit au paragraphe 2 , il résulte de l'analyse du $a$ que $L_{h} / I$ admet un isomorphisme naturel sur $L$ et $L_{h}$ admet la décomposition en somme directe $L_{h}=I+L$. Nous obtenons

Théorème 2. Si une variété kählerienne compacte $W_{n}$ vérifie $c_{1}\left(W_{n}\right) \geqslant 0$, on a pour le scalaire $f>0$ vérifiant (11.1):

$$
\operatorname{dim}_{C} U^{p}(f)=b_{p, 0}\left(W_{n}\right) \leqslant C_{n}^{p}
$$

et par suite $\operatorname{dim}_{C} T^{p} \geqslant b_{p, 0}\left(W_{n}\right)$. Une forme holomorphe non identiquement nulle ne peut s'annuler. L'algèbre $L_{h}$ admet la décomposition en somme directe:

$$
L_{h}=I+L,
$$

où $L$ est une sous-algèbre complexe abélienne de $L_{h}$, de dimension complexe $b_{1,0}\left(W_{n}\right)$, qui laisse invariant chaque élément de $U^{p}(f)$.

Supposons toujours $c_{1}\left(W_{n}\right) \geqslant 0$; si $b_{n, 0}\left(W_{n}\right) \neq 0$, il existe une $n$-forme holomorphe non identiquement nulle sur $W_{n}$ et cette forme ne peut s'annuler nulle part. Par suite $c_{1}\left(W_{n}\right)=0$.

Ainsi si $c_{1}\left(W_{n}\right) \geqslant 0$ n'est pas nulle, on a $b_{n, 0}\left(W_{n}\right)=0$.

\section{Etude de cas particuliers}

a) Supposons que $c_{1}\left(W_{n}\right)$ contienne une 2-forme telle que la forme hermitienne associée $c$, partout négative ou nulle, soit définie négative en un point $z_{0}$ de $W_{n}$. Nous dirons que $c_{1}\left(W_{n}\right) \leqslant 0$ est localement définie négative. 
Si $A$ est un $p$-tenseur holomorphe de $W_{n}$, il appartient à $U^{p}(f)$ et il résulte de (11.4) que $\alpha(A)$ vérifie partout

$$
(Q(c) \alpha(A), f \alpha(A))=0 .
$$

D'après (11.2), cette égalité entraîne qu'au point $z_{0}, \alpha(A)$ donc $A$ s'annulent. Mais $A$ appartenant à $U^{p}(f)$ ne peut, d'après le lemme 2 , s'annuler sans être identiquement nul. Ainsi :

Corollaire 1. Si $c_{1}\left(W_{n}\right) \leqslant 0$ est localement définie négative, il n'existe sur $W_{n}$ aucun p-tenseur holomorphe non identiquement nul. En particulier le plus grand groupe connexe $G$ de transformations holomorphes de $W_{n}$ est réduit à l'identité.

Nous obtenons ainsi une généralisation d'un théorème dû à Nakano et relatif au cas où $c_{1}\left(W_{n}\right)$ est définie négative (c partout définie négative). Sous cette dernière hypothèse, Andreotti et $\mathrm{S}$ Kobayashi [3] ont montré récemment que le groupe de toutes les transformations holomorphes de $W_{n}$ est fini.

b) Supposons maintenant que $c_{1}\left(W_{n}\right)$ contienne une 2-forme telle que la forme hermitienne $c$ associée, partout positive ou nulle, soit définie positive en un point $z_{0}$ de $W_{n}$. Nous dirons alors que $c_{1}\left(W_{n}\right) \geqslant 0$ est localement définie positive.

Si $\alpha \in F^{p}(f)$, il résulte de (12.1) que $\alpha$ vérifie partout:

$$
(Q(c) \alpha, \alpha)=0 \text {. }
$$

D'après (11.2) cette égalité entraîne encore qu'au point $z_{0}, \alpha$ s'annule. Mais, d'après l'hypothèse faite, $\alpha$ est associée à un élément $A$ de $U^{p}(f)$ et ne peut s'annuler sans être identiquement nulle. On voit ainsi que $b_{p, 0}\left(W_{n}\right)=0$ $(p=1, \cdots, n)$. Ainsi :

Corollaire 2. Si $c_{1}\left(W_{n}\right) \geqslant 0$ est localement définie positive, il n'existe sur $W_{n}$ aucune $p$-forme holomorphe non identiquement nulle. En particulier le premier nombre de Betti $b_{1}\left(W_{n}\right)$ est nul.

c) Supposons enfin $c_{1}\left(W_{n}\right)=0$. Les résultats des deux théorèmes fondamentaux des paragraphes 11 et 12 sont simultanément valables. De

$$
\operatorname{dim}_{C} T^{p} \leqslant b_{p, 0}\left(W_{n}\right), \quad \operatorname{dim}_{C} T^{p} \geqslant b_{p, 0}\left(W_{n}\right)
$$

on déduit

$$
\operatorname{dim}_{C} T^{p}=b_{p, 0}\left(W_{n}\right)
$$

Pour $p=1$

$$
\operatorname{dim}_{C} L_{h}=b_{1,0}\left(W_{n}\right)
$$

et $I$ est nul. Ainsi 
Corollaire 3. Si $c_{1}\left(W_{n}\right)=0$, un p-tenseur holomorphe ou une p-forme holomorphe $\neq \equiv 0$ ne peuvent s'annuler. Tout p-tenseur holomorphe est invariant par toute transformation infinitésimale holomorphe et

$$
\operatorname{dim}_{C} T^{p}=b_{p, 0}\left(W_{n}\right) \text {. }
$$

En particulier le plus grand groupe connexe $G$ de transformations holomorphes de $W_{n}$ est abélien et

$$
\operatorname{dim}_{R} G=b_{1}\left(W_{n}\right)
$$

14. Variété de Hodge à $c_{1}\left(W_{n}\right) \geqslant 0$ et localement définie positive

a) Supposons que $W_{n}$ soit une variété de Hodge (variété algébrique) compacte: la 2-forme fondamentale $F$ de $W_{n}$ admet des périodes entières. Si $\chi\left(W_{n}\right)$ est le genre arithmétique de la variété, on a, d'après un résultat classique de Kodaira et Spencer:

$$
\chi\left(W_{n}\right)=\sum_{p=0}^{n}(-1)^{p} b_{p, 0}\left(W_{n}\right) .
$$

Soit $\tilde{W}_{n}$ un revêtement d'ordre $q$ de $W_{n}$ que nous supposerons muni de la métrique et de la 2-forme images réciproques de celles de $W_{n}$. La variété $\tilde{W}_{n}$ est aussi variété de Hodge. En appliquant la version de Hirzeburch du théorème de Riemann-Roch, S. Kobayashi [4] a établi le lemme suivant:

Lemme 4. Soit $\tilde{W}_{n}$ un revêtement d'ordre $q$ d'une variété de Hodge compacte $W_{n}$. On a:

$$
\chi\left(\tilde{W}_{n}\right)=q \chi\left(W_{n}\right)
$$

b) Supposons en outre que $c_{1}\left(W_{n}\right) \geqslant 0$ soit localement définie positive. Il en est alors évidemment de même pour $\tilde{W}_{n}$ et il résulte de (14.1) et du corollaire 2 du paragraphe 13:

$$
\chi\left(W_{n}\right)=1, \quad \chi\left(\tilde{W}_{n}\right)=1 .
$$

On a donc nécessairement $q=1$ d'après le lemme 4 . Le groupe fondamental $\pi_{1}\left(W_{n}\right)$ de la variété envisagée ne peut admetre de sous-groupe propre d'indice fini.

Le premier nombre de Betti de $W_{n}$ étant nul, le groupe $H_{1}\left(W_{n}, Z\right)$ est fini. Le sous-groupe des commutateurs de $\pi_{1}\left(W_{n}\right)$ ne peut que coïncider avec $\pi_{1}\left(W_{n}\right)$ et l'on a :

$$
H_{1}\left(W_{n}, Z\right)=0 \text {. }
$$

Ce raisonnement, dû à Kobayashi, conduit au théorème suivant 
Théorème 3. Soit $W_{n}$ une variété de Hodge compacte telle que $c_{1}\left(W_{n}\right) \geqslant 0$ soit localement définie positive. Pour une telle variété:

$$
H_{1}\left(W_{n}, Z\right)=0 \text {. }
$$

\section{Cas d'une courbure scalaire constante}

Nous nous proposons d'étudier le cas d'une variété kählerienne dont la courbure scalaire $\operatorname{Tr} . R$ est constante.

a) Nous allons d'abord établir le lemme suivant

Lemme 5. Pour toute p-forme holomorphe $\beta$, on a sur une variété kählerienne:

$$
\delta^{\prime} Q(R) \beta=-i(d \operatorname{Tr} . R) \beta
$$

En effet de:

$$
(Q(R) \beta)_{\rho_{1} \cdots \rho_{p}}=\frac{2}{(p-1) !} \varepsilon_{\rho_{1} \cdots \rho_{p}}^{2 \sigma_{2} \cdots \sigma_{p}} R_{\lambda}{ }^{\mu} \beta_{\mu \sigma_{2} \cdots \rho_{p}}
$$

On déduit qu'en coordonnées locales complexes:

$$
\left(\delta^{\prime} Q(R) \beta\right)_{\rho_{2} \cdots \rho_{p}}=-\frac{2}{(p-1) !} \varepsilon_{\nu \rho_{2} \cdots \rho_{p}}^{2 \sigma_{2} \cdots \sigma_{p}} \nabla^{\nu} R_{2}{ }^{\mu} \beta_{\mu \sigma_{2} \cdots \sigma_{p}} .
$$

En distinguant au second membre les termes pour lesquels $\nu=\lambda$ et ceux pour lesquels $\nu=\sigma_{2}, \cdots, \sigma_{p}$, il vient

$$
\left(\delta^{\prime} Q(R) \beta\right)_{\rho_{2} \cdots \rho_{p}}=-2 \nabla^{\nu} R_{\nu}{ }_{\nu} \beta_{\mu \rho_{2} \cdots \rho_{p}}+\frac{2}{(p-2) !} \varepsilon_{\rho_{2} \cdots \rho_{p}}^{2 \sigma_{3} \cdots \sigma_{p}} \nabla^{\nu} R_{\alpha}{ }^{\mu} \beta_{\mu \nu \sigma_{3} \cdots \sigma_{p}} .
$$

Le tenseur de Ricci d'une variété kählerienne vérifie:

$$
\nabla^{\nu} R_{2}{ }^{\mu}=\nabla^{\mu} R_{2}{ }^{\nu}
$$

Comme $\beta_{\mu \nu \sigma_{3} \ldots \sigma_{p}}$ est antisymétrique en $\mu$ et $\nu$ le second terme du membre de droite est nul. D'autre part, d'après l'identité de Bianchi:

$$
2 \nabla^{\nu} R_{\nu}{ }^{\mu}=\nabla^{\mu}(\operatorname{Tr} . R)
$$

et il vient :

$$
\delta^{\prime} Q(R) \beta=-i(d \operatorname{Tr} . R) \beta .
$$

Si Tr. $R=$ const., $\delta^{\prime} Q(R) \beta=0$.

b) Du lemme précédent, on déduit: 
Lemme 6. Soit $A \in T^{p}$ un p-tenseur holomorphe sur la variété kählerienne compacte $W_{n}$ à courbure scalaire constante. Dans la décomposition:

$$
\alpha(A)=d^{\prime} \mu+H \alpha(A)
$$

la p-forme holomorphe $H \alpha(A)$ est à dérivée covariante nulle.

$\mathrm{D}$ 'après la proposition 1 du paragraphe $9, \alpha(A)$ vérifie l'équation:

$$
\Delta \alpha(A)-Q(R) \alpha(A)=0 .
$$

On en déduit:

$$
\begin{aligned}
<\Delta d^{\prime} \mu-Q(R) d^{\prime} \mu, d^{\prime} \mu> & =-<\Delta H \alpha(A)-Q(R) H \alpha(A), d^{\prime} \mu> \\
& =<Q(R) H \alpha(A), d^{\prime} \mu>
\end{aligned}
$$

soit

$$
<\Delta d^{\prime} \mu-Q(R) d^{\prime} \mu, d^{\prime} \mu>=\left\langle\delta^{\prime} Q(R) H \alpha(A), \mu>.\right.
$$

Mais d'après le lemme 5 , le second membre est nul. De la proposition $1 \mathrm{du}$ paragraphe 9, il résulte que la $p$-forme $d^{\prime} \mu$ et, par suite, la $p$-forme $H \alpha(A)$ sont associées à des $p$-tenseurs holomorphes; $H \alpha(A)$ vérifie donc les relations:

$$
\nabla_{\lambda}\{H \alpha(A)\}_{\rho_{1} \cdots \rho_{p}}=0, \quad \nabla_{\bar{\lambda}}\{H \alpha(A)\}_{\rho_{1} \cdots \rho_{p}}=0
$$

et est ainsi à dérivée covariante nulle.

c) Une variété riemannienne est dite irréductible si son groupe d'holonomie connexe est irréductible (dans le réel). Nous allons établir le lemme suivant :

Lemme 7. Soit $W_{n}$ une variété kählerienne compacte telle que $c_{1}\left(W_{n}\right) \geqslant 0$, sans être nulle. Si $W_{n}$ est irréductible et à courbure scalaire constante, on a:

$$
b_{p, 0}\left(W_{n}\right)=0 \quad(p=1, \cdots, n) .
$$

En effet, d'après le raisonnement conduisant au théorème 2 , toute $p$-forme holomorphe $\beta$ de $W_{n}$ peut s'écrire $\beta=H \alpha(A)$, où $A$ est un $p$-tenseur holomorphe. Il résulte du lemme 6 que, sous les hypothèses faites, toute $p$-forme holomorphe $\beta$ de $W_{n}$ est à dérivée covariante nulle, donc vérifie simultanément $\Delta \beta=0, \Delta \beta-Q(R) \beta=0$ et par suite $Q(R) \beta=0$.

On en déduit:

$$
(Q(c) \beta, \beta)+2 \nabla_{2}\left(\nabla_{\bar{\mu}} \log f \beta^{i \sigma_{2} \cdots \sigma_{p}} \beta_{\sigma_{2} \cdots \sigma_{p}}\right)=0 .
$$

Par intégration sur $W_{n}$, il vient:

$$
\langle Q(c) \beta, \beta>=0 .
$$


$(Q(c) \beta, \beta)$ étant nécessairement $\geqslant 0$, on en déduit:

$$
(Q(c) \beta, \beta)=0
$$

soit en coordonnées locales:

$$
c_{\lambda \bar{\mu}} \beta^{2 \sigma_{2} \cdots \sigma_{p}} \beta_{\sigma_{2} \cdots \sigma_{p}}^{\bar{\mu}}=0 .
$$

Si $\beta \not \equiv 0$ est une $p$-forme holomorphe, il résulte de l'irréductibilité de $W_{n}$ :

$$
\beta^{2 \sigma_{2} \cdots \sigma_{p}} \beta_{\sigma_{2} \cdots \sigma_{p}}^{\bar{\mu}}=a g^{\lambda \bar{\mu}} \quad(a=\text { const. } \neq 0)
$$

puisque le premier membre est à dérivée covariante nulle. De (15.2), il résulterait alors Tr. $c \equiv 0$, ce qui est contraire aux hypothèses du lemme. On en déduit $b_{p, 0}\left(W_{n}\right)=0(p=1, \cdots n)$.

b) Supposons que $W_{n}$, variété de Hodge, satisfasse aux hypothèses du lemme 7 . Le raisonnement du paragraphe 14 s'applique sans modification à la situation présente et l'on a (voir [4])

Théorème 4. Soit $W_{n}$ une variété de Hodge compacte telle que $c_{1}\left(W_{n}\right) \geqslant 0$, sans être nulle. Si $W_{n}$ est irréductible et à courbure scalaire constante, on a:

$$
H_{1}\left(W_{n}, Z\right)=0 \text {. }
$$

\section{Bibliographie}

[1] Y. Akizuki \& S. Nakano, Note on Kodaira-Spencer's proof of Lefschetz theorems, Proc. Japan Acad. 30 (1954) 266-272.

[2] S. S. Chern, Characteristic classes of Hermitian manifolds, Ann. of Math. 47 (1948) 85-121.

[3] S. Kobayashi, On the automorphism group of a certain class of algebraic manifolds, Tôhoku Math. J. 11 (1959) 184-190.

[4] - On compact Kähler manifolds with positive definite Ricci tensor, Ann. of Math. 74 (1961) 570-573.

[5] S. Kobayashi \& K. Nomizu, Foundations of differential geometry, Vol I, Interscience, New York, 1963.

[6] K. Kodaira, On a differential-geometric method in the theory of analytic stacks, Proc. Nat. Acad. Sci. U.S.A. 39 (1953) 1268-1273.

[7] K. Kodaira \& D. C. Spencer, On arithmetic genera of algebraic varieties, Proc. Nat. Acad. Sci. U.S.A. 39 (1953) 641-649.

[8] A. Lichnerowicz, Théorie globale des connexions et des groupes d'holonomie, Cremonese, Rome, 1955; Géométrie des groupes de transformations, Dunod, Paris, 1958.

[9] - Isométries et transformations analytiques d'une variété kählérienne compacte, Bull. Soc. Math. France 87 (1959) 427-437.

[10] - Sur certaines variétés kählériennes compactes, C. R. Acad. Sci. Paris 263 (1966) 570-575.

[11] - Variétés complexes et tenseur de Bergmann, Ann. Inst. Fourier (Genoble) 15 (1965) 345-407. 
